\title{
ANÁLISE DE SISTEMAS DE CORROSÃO EM DIFERENTES MEIOS
}

\author{
João Paulo Marcolino Beda Campos \\ Graduando em Engenharia Mecânica / ISECENSA/ RJ \\ jpcampos@technip.com
}

\section{Thiago Gomes Rodrigues dos Santos}

Graduando em Engenharia Mecânica / ISECENSA/RJ

tgrgomes@live.com

\section{Geanni Barbosa da Silveira e Silva}

Mestre em Engenharia e Ciências dos Materiais/ UENF/ RJ

Professora Engenharia Mecânica e Engenharia de Produção / ISECENSA/ RJ

gi_silveira@hotmail.com

\section{Mário Lucas Santana Silva}

Doutor em Engenharia e Ciências dos Materiais/ UENF/ RJ

Professor Engenharia Mecânica e Engenharia de Produção / ISECENSA/ RJ

mariolucas@ymail.com

\section{RESUMO}

Todos os metais e ligas metálicas estão sujeitos a sofrer corrosão. A devota necessidade de aumentar a resistência dos materiais à corrosão se dá pelo fato que as empresas estão em crescente preocupação em reduzir seus custos com disperdício de materiais, possíveis acidentes, além de minimizar o impacto ambiental que a corrosão causa. $\mathrm{O}$ aumento da durabilidade dos materiais fundamenta o constante estudo das formas de corrosão. Afim de que suas características sejam cada vez mais conhecidas e suas influencias na perda de propriedades dos materiais sejam reduzidas. $\mathrm{O}$ projeto teve como objetivo, analisar $\mathrm{o}$ comportamemto de um aço carbono exposto a três meios corrosivos, a saber, água doce, água salgada e solo. Diante da crescente busca pelo estudo da corrosão para a integridade de instalações metálicas expostas a ambientes presentes na indústrias em geral, este projeto foi elaborado para investigar algumas questões que se apresentaram interessantes para a prática do monitoramento da corrosão com uso de corpos de prova de materiais largamente empregados neste meio.

Palavras-chave: Corrosão; Monitoramento; Aço carbono. 


\begin{abstract}
All metals and alloys are subject to corrosion. The devout need to increase the corrosion resistance of materials is caused by the fact that companies are growing concern to reduce their costs with waste of materials, possible accidents and minimize the environmental impact of corrosion cause. Increased durability of the materials underlying the constant study of the forms of corrosion. In order that its features are increasingly popular and their influence in the loss of material properties are reduced. The project aimed to analyze the behavior an exposed carbon steel three corrosive environment, namely fresh water, salt water and soil. Given the growing demand for the study of corrosion to the integrity of metal installations exposed to environments present on industries in general, this project was designed to investigate some issues that appeared interesting for the practice of the corrosion monitoring with use of specimens of materials widely used in this environment.
\end{abstract}

Keywords: Corrosion; Monitoring; Carbon steel.

\title{
INTRODUÇÃO
}

A corrosão causa problemas frequentes nos mais diversos setores onde são empregados, prejudicam a durabilidade e desempenho de estruturas, máquinas, equipamentos e ferramentas. Seu estudo é imprescindível para evitar impactos econômicos desnecessários com manutenção indesejada e de segurança na sociedade. Como forma de minimizar estes prejuízos, são pesquisadas formas de proteger e impedir a ação corrosiva provocada pelo meio nos metais. Para que não haja falhas nas condições finais do produto em seu uso, ainda na fase de projeto, deve-se levar em consideração fatores relacionados à deterioração metálica, tais como a seleção de materiais mais resistentes e aumento da espessura; utilização de revestimentos, inibidores de corrosão, técnicas eletroquímicas; inspeção, manutenção e reparação. Outro aspecto importante da corrosão está relacionado com a conservação das reservas de minérios. Tendo em vista a permanente destruição dos materiais metálicos pela corrosão, há necessidade de uma produção adicional desses materiais para repor o que foi deteriorado, e esta parcela é muito significativa. A literatura mais antiga reporta que $25 \%$ da produção mundial de aço têm esta finalidade (GENTIL, 2012).

Dentre as formas de corrosão mais comuns em aço carbono, a corrosão por pites ganha destaque. A corrosão por pites consiste na formação de cavidades, geralmente, de pequeno diâmetro e maior profundidade, que podem chegar a perfurar toda a espessura do material atacado, com pouca ou nenhuma perda uniforme, por isso, muito destrutiva e perigosa. A causa geral da corrosão por pites é a existência de pequenas áreas no material, altamente anódicas em relação às áreas catódicas adjacentes, formando as chamadas "pilhas passivo-ativa" (SILVEIRA, 2014).

Em alguns processos existem muitas dificuldades em caracterizar as mais variadas formas de cavidades, entretanto, deve-se considerar que a importância maior é a determinação das dimensões dessas cavidades, a fim de se verificar a extensão do processo corrosivo. De forma bem específica é aconselhável caracterizar a corrosão por pites de acordo com sua densidade, área e profundidade (SILVEIRA, 2014).

Conhecendo os tipos de corrosão e compreendendo seus mecanismos e suas causas, torna-se possível tomar medidas para prevenir a ocorrência desses fenômenos. Por exemplo, podemos alterar a natureza do ambiente, selecionar um material que seja relativamente não reativo e/ou proteger o material contra uma deterioração apreciável (CALLISTER, 1999).

Este trabalho tem o objetivo de investigar a corrosão de um aço carbono, onde foram avaliadas e estudadas as formas de corrosão em três ambientes: água do mar, água doce e solo. 


\section{METODOLOGIA}

Foram utilizados 12 corpos de prova (CP's) para o experimento, onde metade deles foram lixados e o restante permaneceu com acabamento superficial da forma com que foi adquirido. Os mesmos foram distribuídos da seguinte forma: lixados - 2 em água do mar, 2 em água de rio e $2 \mathrm{em}$ solo. Os demais não lixados foram distribuídos de igual forma. A partir deste comparativo pode-se perceber as diferentes degradações e constatar a relação da corrosão com o tipo de acabamento superficial.

Foi realizada a imersão dos CP's nos meios para análise, com duração de aproximadamente 3 meses. Cada corpo de prova foi imerso em embalagens do tipo PET com volume aproximado de 2 litros. Após o ensaio foi realizada a decapagem química dos cupons para a retirada do produto de corrosão depositado, para tal procedimento foi utilizada a norma NBR 6210 (ABNT) como referência. E por fim, o ensaio visual para a detecção da forma de corrosão apresentada.

Por motivos de discrição e confidencialidade relataremos o laboratório como " $\mathrm{X}$ " e cada corpo de prova terá a seguinte denominação:

Amostra lixada - AML

Amostra sem lixar - AMSL

\section{Material utilizado}

O material analisado foi um aço com teor de $0,60 \%$ a $0,75 \%$ de carbono usado na fabricação de tubos flexíveis para extração de petróleo em plataformas offshore, que tem sua composição química e propriedades mecânicas mais detalhadas no quadro abaixo (quadro I).

Quadro I: Composição química e propriedades mecânicas do material utilizado.

\begin{tabular}{|l|l|l|l|l|c|c|}
\hline \multicolumn{7}{|c|}{ COMPOSIÇÃO QUíMICA } \\
\hline \multicolumn{1}{|c|}{$\%$} & $\mathrm{C}$ & $\mathrm{Si}$ & $\mathrm{Mn}$ & $\mathrm{Al}$ & $\mathrm{S}$ & $\mathrm{P}$ \\
\hline Min. & 0.60 & 0.10 & 0.50 & - & - & - \\
\hline Max. & 0.75 & 0.35 & 1.00 & 0.070 & 0.030 & 0.030 \\
\hline \multicolumn{7}{|c|}{ PROPRIEDADES MECÂNICAS } \\
\hline TS & Mpa & Tensão de alongamento a 0,2\% & $\geq 1080$ \\
\hline UTS & Mpa & Força de tensão & $1200 \leq$ UTS 1280 \\
\hline A & $\%$ & Elongação & $\geq 8 \%$ \\
\hline Dureza & HV 30 & Vickers 30 Kg ou equivalente & Para informação \\
\hline
\end{tabular}

Fonte: Laboratório "X".

As dimensões podem ser verificadas no quadro II.

Quadro II: Medidas de comprimento, largura e espessura dos CP's.

\begin{tabular}{|c|c|c|}
\hline Amostras sem lixamento & Amostras lixadas & Ambiente \\
\hline AMSL 1: $80 \mathrm{~mm} \times 20 \mathrm{~mm} \times 5 \mathrm{~mm}$ & AML 1: $85 \mathrm{~mm} \times 20 \mathrm{~mm} \times 5 \mathrm{~mm}$ & Água do mar \\
\hline AMSL 2: $85 \mathrm{~mm} \times 20 \mathrm{~mm} \times 5 \mathrm{~mm}$ & AML 2: $85 \mathrm{~mm} \times 20 \mathrm{~mm} \times 5 \mathrm{~mm}$ & Água do mar \\
\hline AMSL 3: $85 \mathrm{~mm} \times 20 \mathrm{~mm} \times 5 \mathrm{~mm}$ & AML 3: $85 \mathrm{~mm} \times 20 \mathrm{~mm} \times 5 \mathrm{~mm}$ & Água do rio \\
\hline AMSL 4: $85 \mathrm{~mm} \times 20 \mathrm{~mm} \times 5 \mathrm{~mm}$ & AML 4: $84 \mathrm{~mm} \times 20 \mathrm{~mm} \times 5 \mathrm{~mm}$ & Água do rio \\
\hline AMSL 5: $84 \mathrm{~mm} \times 20 \mathrm{~mm} \times 5 \mathrm{~mm}$ & AML 5: $84 \mathrm{~mm} \times 20 \mathrm{~mm} \times 5 \mathrm{~mm}$ & Solo \\
\hline AMSL 6: $86 \mathrm{~mm} \times 20 \mathrm{~mm} \times 5 \mathrm{~mm}$ & AML 6: $86 \mathrm{~mm} \times 20 \mathrm{~mm} \times 5 \mathrm{~mm}$ & Solo \\
\hline
\end{tabular}

Fonte: Medidas realizadas no Laquibio (Instituto Superior de Ensino Nossa Senhora Auxiliadora, ISECENSA) pelos autores. 


\section{Meios utilizados}

Será descrita a localização dos meios utilizados:

Água do mar coletada em Marataízes E.S. (Latitude: $21^{\circ} 3^{\prime} 43.84 " \mathrm{~S}$; Longitude: $40^{\circ} 49^{\prime} 59.00^{\prime \prime O}$ ), água do rio Muqui do Sul (Latitude: $21^{\circ} 0^{\prime} 1.24 " \mathrm{~S}$; Longitude: $\left.41^{\circ} 28^{\prime} 48.29^{\prime \prime O}\right)$ e solo coletado em Mimoso do Sul E.S (Latitude: $21^{\circ} 1$ 1'12.06"S; Longitude: 41³0'16.96"O).

\section{RESULTADOS}

Assim que os cupons foram retirados dos meios, foi realizada a decapagem dos mesmos e foram pesados. A decapagem foi realizada com base na norma 6210 NBR da ABNT, onde foi utilizada uma solução de $15 \%$ de ácido sulfúrico e o cupom foi mantido imerso por 10 minutos. Após esse processo as amostras foram lavadas com água corrente fraca para retirada dos resíduos do ácido. No quadro III serão expostos os pesos registrados (inicial e final) para verificação de perda de massa, caracterizada pela corrosão uniforme.

Quadro III: Pesagem das amostras segue abaixo.

\begin{tabular}{|c|c|c|c|}
\hline Nome & Peso inicial & Peso após decapagem & Perda de massa (\%) \\
\hline AMSL 1 & $62,5915 \mathrm{~g}$ & $61,9239 \mathrm{~g}$ & 1,06660 \\
\hline AMSL 2 & $66,5415 \mathrm{~g}$ & $65,9002 \mathrm{~g}$ & 0,96376 \\
\hline AMSL 3 & $66,4448 \mathrm{~g}$ & $65,3521 \mathrm{~g}$ & 1,64452 \\
\hline AMSL 4 & $65,9042 \mathrm{~g}$ & $64,7328 \mathrm{~g}$ & 1,77743 \\
\hline AMSL 5 & $65,8519 \mathrm{~g}$ & $65,5584 \mathrm{~g}$ & 0,44570 \\
\hline AMSL 6 & $66,4491 \mathrm{~g}$ & $66,3052 \mathrm{~g}$ & 0,21656 \\
\hline AML 1 & $66,2121 \mathrm{~g}$ & $65,3316 \mathrm{~g}$ & 1,32982 \\
\hline AML 2 & $66,5626 \mathrm{~g}$ & $65,8305 \mathrm{~g}$ & 1,09987 \\
\hline AML 3 & $66,1192 \mathrm{~g}$ & $65,0817 \mathrm{~g}$ & 1,56913 \\
\hline AML 4 & $65,7170 \mathrm{~g}$ & $64,8781 \mathrm{~g}$ & 1,27653 \\
\hline AML 5 & $65,9361 \mathrm{~g}$ & $65,6383 \mathrm{~g}$ & 0,45165 \\
\hline AML 6 & $66,2431 \mathrm{~g}$ & $66,0525 \mathrm{~g}$ & 0,28773 \\
\hline
\end{tabular}

Quadro III: Comparativos de peso das amostras e porcentagem de perda de massa.

Para uma melhor visualização e entendimento dos resultados obtidos, será apresentada uma série de gráficos em barra para comparação dos pesos (gráfico I). 


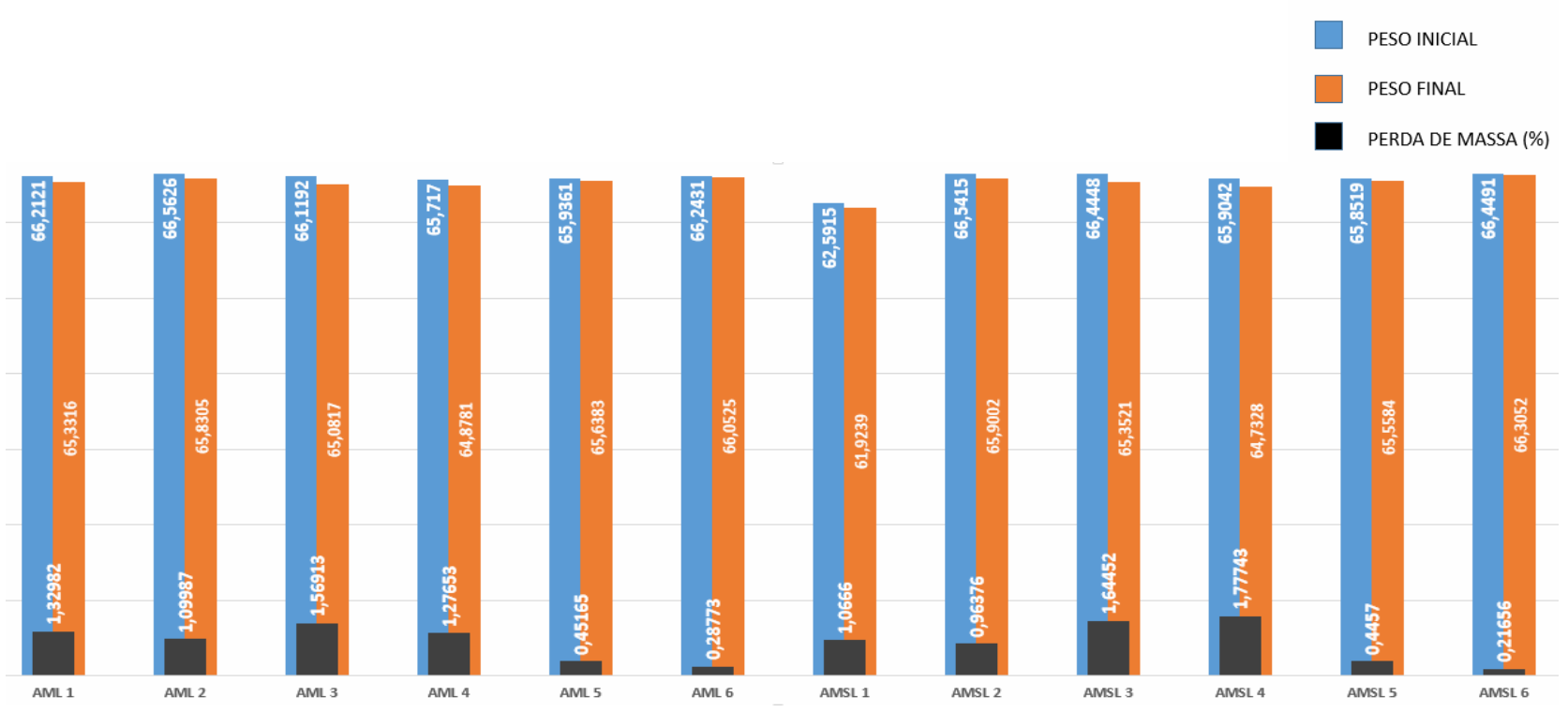

Gráfico I: comparativo de peso e porcentagem de perda de massa.

Pode-se perceber maior perda de massa na amostra AMSL 4, em seguida da AMSL3, ambas imersas em água do rio. E a amostra menos afetada foi a AML 6, imerso em solo. Isso se deve ao fato de que os materiais metálicos em geral em contato com água tendem a sofrer maior corrosão em decorrência de agregados metálicos, que se formam pela ação de íons metálicos sob influência de moléculas de água (WOLYNEC, 2003), bem como a presença de alguns contaminantes, tais como: gases e sais dissolvidos, matéria orgânica de origem animal e vegetal, bactérias, limos, algas e sólidos em suspensão (GENTIL, 2012). O quadro IV demonstra o $\mathrm{pH}$ e a temperatura dos meios para cada amostra (com e sem lixamento). Como o ensaio foi realizado em laboratório, houve pouca diferença de temperatura entre os meios, como pode ser observado.

Quadro IV: $\mathrm{pH}$ e a temperatura dos meios.

\begin{tabular}{|c|c|c|}
\hline Nome & $\mathrm{pH}$ & Temperatura ${ }^{\circ} \mathrm{C}$ \\
\hline AML 1 & 7,1 & 26,2 \\
\hline AML 2 & 7,6 & 27,2 \\
\hline AML 3 & 6,9 & 25,6 \\
\hline AML 4 & 8,43 & 25,6 \\
\hline AML 5 & 5,6 & 26,3 \\
\hline AML 6 & 5,2 & 26,3 \\
\hline AMSL 1 & 7,5 & 26,1 \\
\hline AMSL 2 & 7,6 & 25,9 \\
\hline AMSL 3 & 7,8 & 25,8 \\
\hline AMSL 4 & 7,8 & 25,9 \\
\hline AMSL 5 & 5,3 & 26,3 \\
\hline AMSL 6 & 5,6 & 26,2 \\
\hline
\end{tabular}


Através de um ensaio visual pode-se identificar que a corrosão que mais se destacou no material foi a corrosão por pites, como ilustra a figura 1 .

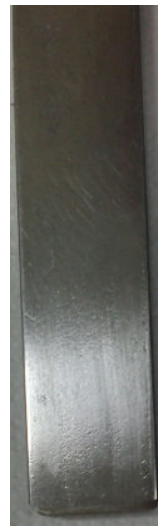

(a)

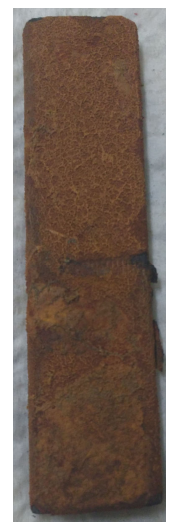

(b)

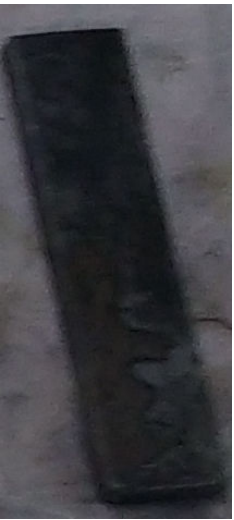

(c)

Figura 1: AML 1 - (a) após lixamento, (b) após ensaio de corrosão e (c) após decapagem química.

\section{CONSIDERAÇÕES FINAIS}

As amostras tanto lixadas quanto sem lixamento imersas em água do mar e do rio tiveram uma deterioração bem mais elevada em comparação com o solo.

Observou-se que as amostras lixadas e sem lixamento não tiveram perda de massa significativa comparadas entre si, porém em concordância com a bibliografia o acabamento superficial afeta diretamente a velocidade do processo corrosivo, não muito evidente nesse caso pelo material não ter nenhuma proteção em sua superfície.

Comparando todos os meios, a maior perda de massa apresentou-se na água do rio e não na água de mar como esperado para este tipo de aço, visto que o material estudado é utilizado em ambiente offshore constantemente, o que comprova sua eficiência para este meio.

A corrosão mais abrangente nas amostras se deu na forma de pites.

\section{REFERÊNCIAS BIBLIOGRÁFICAS}

ABNT 6210 NBR. Corrosão atmosférica - Materiais metálicos - Preparo, limpeza e determinação da taxa de corrosão de corpos-de-prova em ensaios de corrosão, ISBN 978-85-07-01103-3, 9p., 2008.

CALLISTER JR., W.D. Ciência e Engenharia de Materiais: Uma Introdução, 5 Edição. Editora LTC, 2000. GENTIL, V., Corrosão. $6^{\mathrm{a}}$ edição, Rio de Janeiro: LTC, 2012.

SILVEIRA, G. B. et al. Microscopia Óptica e Microscopia Confocal como Técnicas Comparativas para Caracterização de Pites em Cupons de Corrosão. In: INTERCORR., 2014, Fortaleza, CE.

WOLYNEC, S. Técnicas Eletroquímicas em Corrosão. Editora da USP, São Paulo, 2003. 